\title{
Attention modulates incidental memory encoding of human movements
}

\author{
Shiau-Chuen Chiou $^{1,2}$ (1)
}

Received: 19 July 2021 / Accepted: 25 January 2022 / Published online: 28 February 2022

(C) The Author(s) 2022

\begin{abstract}
Attention has been shown to enhance the processing of task-relevant information while suppressing the processing of task-irrelevant information. However, it is less clear whether this attentional modulation exists when there is an intrinsic dependence between task-relevant and task-irrelevant information, such as the dependence of temporal processing on spatial information. In this study, we used complex whole-body movement sequences to investigate the extent to which the taskirrelevant spatial information (trajectory) is processed when only the temporal information (rhythm) is in focus. Moreover, we examined, if the task-irrelevant spatial information is "co-selected" with the target temporal information as predicted by the intrinsic spatiotemporal dependence, whether task-driven attention that is actively directed to spatial information provides extra benefits. Through a two-phase experiment (an incidental encoding phase followed by a surprise memory test phase), we found that the task-irrelevant spatial information was not only perceived but also encoded in memory, providing further evidence in support of a relatively automatic co-selection of spatial information in temporal processing. Nevertheless, we also found that movements whose trajectories were intentionally attended to during the encoding phase were recognized better in the test phase than those that were not, indicating a further modulation from attention on incidental memory encoding and information processing.
\end{abstract}

Keywords Attention $\cdot$ Memory $\cdot$ Spatiotemporal dependence $\cdot$ Whole-body movement

\section{Introduction}

Attention provides a means of selecting information from the environment that is most relevant to current behavioral goals. Attention can also facilitate sensory processsing (Corbetta et al. 1990; Reynolds and Chelazzi 2004; Yantis and Serences 2003) and enhance memory encoding (Aly and Turk-Browne 2016; Chun and Turk-Browne 2007; Gazzaley and Nobre 2012; Uncapher and Rugg 2009). Although it has been well established that the attended information, either

Handling Editor: Anna Belardinelli (Honda Research Institute Europe); Reviewers: Two researcher who prefer to remain anonymous.

Shiau-Chuen Chiou

shiau-chuen.chiou@uni-bielefeld.de

1 Neurocognition and Action Research Group, Center for Cognitive Interaction Technology (CITEC), Bielefeld University, Inspiration 1, 33619 Bielefeld, Germany

2 Faculty of Psychology and Sports Science, Bielefeld University, Bielefeld, Germany in a particular location or with a specific feature, will be processed more efficiently and better remembered, it is less clear whether the unattended or task-irrelevant information can be excluded from processing, and if yes, to what extent and in which stage (i.e., early perceptual stage or late cognitive stage) (see Driver 2001 for a review).

The load theory of selective attention (Lavie 1995, 2005, 2010; Lavie et al. 2004; see Murphy et al. 2016 for a recent review) suggests that the extent to which taskirrelevant distractors are perceived depends on both perceptual load and cognitive load of the current task. When the perceptual load is high, no spare attentional resources are available for distractors, resulting in performance that is consistent with "early-selection" view of attention (e.g., Broadbent 1958; Treisman 1969). On the contrary, when the perceptual load is low, the spare capacity involuntarily "spills over" to process task-irrelevant information, and thus the suppression of distractors would rely on "late selection", which is supposed to prevent the perceived task-irrelevant information from entering awareness or gaining control over behavior (e.g., Deutsch and Deutsch 
1963; Duncan 1980). Since to actively maintain stimulusprocessing priorities requires executive cognitive control, high cognitive load of the current task, such as from working memory, may impair the efficiency of late selection (de Fockert et al. 2001; Lavie 2010).

Although the load theory has been influential since its proposal, there has been evidence showing that perceptual load is not the only factor that determines the selection processes (see Benoni and Tsal 2013; Khetrapal 2010 for critical reviews). Factors such as perceptual grouping or object-based attention may also affect the degree of distractor processing. Specifically, it has been shown that when task-relevant and task-irrelevant information pertain to the same object or perceptual group (through Gestalt cues like continuation or connectedness), the corresponding objectbased attention will override the effect of perceptual load and dominate the processing of task-irrelevant information likely through the mechanism of "attentional spreading" (Chen 2003; Cosman and Vecera 2012; Richard et al. 2008). Namely, the task-irrelevant information will be "co-selected" with the target information and subject to attentional modulation irrespective of perceptual load. Note that the inherent properties of a stimulus may further influence how effective irrelevant features of an attended object can be suppressed. For example, Mayer and Vuong (2012) showed that unattended color or motion did not affect perceptual discrimination of attended shape, while unattended shape affected the processing of attended motion. The finding is consistent with the "shape bias" in object recognition (Biederman 1987) and suggests that the shape might be processed automatically (i.e., difficult to suppress) due to its dominant role in object recognition.

A similar asymmetric relationship has been found in the processing of spatial and temporal information (Casasanto and Boroditsky 2008; Casasanto et al. 2010; Dormal and Pesenti 2013; Santiago et al. 2011; Starr and Brannon 2016). For example, Casasanto and Boroditsky (2008) asked participants to reproduce spatial displacement or temporal duration of a visually presented stimulus (i.e., a growing line or a moving dot). Results revealed that participants' reproduction of duration was consistently interfered by irrelevant spatial information, while their reproduction of displacement remained precise regardless of irrelevant temporal information. The finding indicates that participants were unable to ignore task-irrelevant spatial information when processing the corresponding temporal information, but not vice versa. This line of research suggests that temporal representation may be intrinsically dependent on spatial representation (Casasanto and Boroditsky 2008; Casasanto et al. 2010) or that the spatial information may be processed more automatically than temporal information due to its higher salience in the visual modality (Dormal and Pesenti 2013; Santiago et al. 2011; Starr and Brannon 2016).
Previous research on asymmetric space-time interdependence has provided evidence in support of a relatively automatic co-selection of task-irrelevant spatial information in temporal processing. However, since most research so far has been using simple stimuli (e.g., lines or dots), it is unclear whether this finding can be generalized to more complex stimuli such as whole-body human movements or actions. For one thing, increased complexity of a stimulus may impose additional demand on both perceptual and cognitive systems and thus change the way the stimulus is processed; for another thing, the possibility of forming an abstract higher-order representation from complex stimuli may decrease the reliance of temporal representation on spatial information.

As human movements unfold over time, the spatial and temporal information can be viewed as intrinsically integrated at an early stage of processing. For example, a continuous movement percept can be formed by integrating the "snapshots" of body shapes over time (Giese and Poggio 2003; Lange et al. 2006). At a higher level, temporal information can also be defined as the change of spatial information over time, such as speed (i.e., the distance travelled along a trajectory divided by elapsed time) or rhythm (i.e., the structure of temporal durations conveyed through a sequence of movements). Based on this definition, it is reasonable to hypothesize that the processing of temporal information would rely, to some extent, on spatial information as being second-order features (but not vice versa), and that the task-irrelevant spatial information would still be processed when only the temporal information is in focus. What is less clear, however, is to what extent this co-selection might occur. Does it merely occur in the perceptual level, or it might go beyond that into cognitive processes such as memory? It is also unclear, if the spatial information, albeit task-irrelevant, is likely to be co-selected with the target temporal information, whether additional attention that is actively directed to the spatial information (due to task relevance) provides extra benefits.

In the current study, we used complex whole-body movement sequences as visual stimuli to investigate the extent to which the task-irrelevant spatial information (movement trajectory) is processed when only the temporal information (movement rhythm) is task-relevant. We also examined how the active attention to spatial information (as opposed to unintended co-selection) might influence its level of processing as measured by the long-term retention in memory. We designed a two-phase experiment including an incidental encoding phase and a surprise memory test phase. During the encoding phase, participants performed a change detection task (same/different judgment) on whole-body movement sequences with two foci of attention: (1) Temporal-only and (2) Both. In the Temporal-only condition, only temporal changes could occur and thus the spatial information was 
task-irrelevant; while in the Both condition, the change could occur in either spatial or temporal domain and thus both types of information were task-relevant. Note that the "change" we referred to in the current study was the change of the whole sequence (i.e., movement trajectory or movement rhythm of the sequence, see "Method") rather than the change of a single unit of the sequence.

Importantly, to better probe the effect of task-driven attention on spatial processing as opposed to the effect of "coselection" or "attentional spreading", in which spatial information is automatically processed with the target temporal information, we used a stimulus set of which the processing demand of spatial information was low (i.e., participants' sensitivity to spatial changes was high) to minimize the task-driven redistribution of attentional resources between spatial and temporal domains. Since the processing demand of temporal information was relatively high (i.e., participants' sensitivity to temporal changes was relatively low), it is assumed that any re-allocation of attentional resources from temporal to spatial processing in the Both condition would impair temporal performance, as no spare capacity was available. This might further result in qualitative changes of processing strategy between the Temporal-only and Both conditions, making the effect of attentional focus less comparable.

The encoding phase was followed by a 10-min break and then the surprise memory test phase wherein participants performed a recognition task (old/new judgment) on movement segments extracted from observed or new sequences. Note that the "surprise" means that participants were not informed about the memory test in advance and thus had no incentive to actively memorize the movements observed in either the Temporal-only or the Both condition. We used the recognition performance as a key indicator to examine whether the task-irrelevant spatial information is encoded in memory and whether the additional task-driven attention on spatial information during the encoding phase provides extra benefits to its long-term retention. Given the dependence of temporal processing on spatial information observed in previous research, we predicted that the task-irrelevant spatial information would be co-selected and processed. Nevertheless, active attention might further enhance the processing of the attended information.

\section{Method}

\section{Participants}

Forty participants were recruited for the experiment. Two were excluded from analyses due to below-chance performance (out of 2 standard deviations from the group mean), leaving a final sample of 38 participants ( 23 female; aged
18-40 years, $M=24.8, \mathrm{SD}=4.0)$. A minimum sample size of 34 was determined based on a power analysis (using $\mathrm{G}^{*}$ Power 3.1; Faul et al. 2007) to provide a power of 0.80 at an alpha level of 0.05 to detect medium effects $(d=0.5)$ for within-subjects comparisons of performance under two experimental conditions (Temporal-only vs. Both). Participants were naïve to the purpose and the design of the experiment. None of them have viewed the experimental materials before.

Participants' experiences in dance, music, and sport were evaluated by a questionnaire and reported here as expertise indexes (0: No experience, 1: Beginner, 2: Intermediate amateur, 3: Advanced amateur, 4: Professional) of 0.5 $(\mathrm{SD}=0.8), 0.7(\mathrm{SD}=0.8)$, and $1.4(\mathrm{SD}=1.1)$, respectively, defined by both the training length and skill level. ${ }^{1}$ No professionals were recruited. Participants signed informed consent prior to the experiment and received $€ 8$ per hour for their participation. The study was conducted in accordance with the ethical principles stated within the declaration of Helsinki (1964) and was approved by the Ethics Committee of Bielefeld University.

\section{Stimuli and apparatus}

Thirty-eight whole-body movement sequences selected from a stimulus pool originally created for another study (unpublished data $)^{2}$ were used in the current experiment, of which 30 sequences were used in the incidental encoding phase and all 38 sequences were used in the surprise memory test phase (see "Procedure" and Table 1).

\section{Movement design}

Each sequence started with a relaxed standing pose (i.e., feet were shoulder width apart and arms were at the sides of the body), followed by four movement units. A movement unit was defined as a coordinated whole-body movement that can be performed with a bell-shaped velocity profile (i.e., accelerating till the midpoint of the movement and then decelerating) (Abend et al. 1982). ${ }^{3}$ Thus, each movement unit had a

\footnotetext{
1 The expertise index (0-4) is defined as the following: 0: No experience, 1: Beginner (skill level $=1$ or 2 in a five-point scale, or skill level $>2$ but training length $<3-5$ years), 2 : Intermediate amateur (skill level $=3$ and training length $\geq 3-5$ years, or skill level $>3$ but training length $<6$ years), 3: Advanced amateur (skill level $=4$ and training length $\geq 6$ years), 4 : Professionals (skill level $=5$ and training length $\geq 6$ years).

${ }^{2}$ A total of 44 whole-body movement sequences were originally designed and recorded for another study conducted by the author, which is currently in preparation and not yet published.

3 There were a few exceptions, such as a jump or a circular movement, that were not performed with a bell-shaped velocity profile. Those movements were included into the stimulus set only if a clear
} 
Table 1 Trial distribution and its relation to movement sequences

\begin{tabular}{|c|c|c|c|c|c|c|c|}
\hline & \multirow[t]{3}{*}{$\begin{array}{l}\text { Number of } \\
\text { sequences }\end{array}$} & \multirow{2}{*}{\multicolumn{3}{|c|}{$\begin{array}{l}\text { Incidental encoding phase: change } \\
\text { detection task }\end{array}$}} & \multirow{2}{*}{\multicolumn{3}{|c|}{$\begin{array}{l}\text { Surprise memory test phase: } \\
\text { recognition task }\end{array}$}} \\
\hline & & & & & & & \\
\hline & & Same & Different & Total & 1-unit & 2-unit & Tota \\
\hline Temporal-only & 12 & 24 & 24 & 48 & 24 & 12 & 36 \\
\hline Both $_{\text {temporal }}$ & 12 & 24 & 24 & 48 & 24 & 12 & 36 \\
\hline Both $_{\text {filler }}$ & 6 & 24 & 24 & 48 & 12 & 6 & 18 \\
\hline New & 8 & & & & 16 & 8 & 24 \\
\hline Total & 38 & & & & 76 & 38 & 114 \\
\hline
\end{tabular}

In the Both condition of the change detection task, sequences with potential spatial changes, i.e., spatial fillers $\left(\right.$ Both $\left._{\text {filler }}\right)$, were displayed twice as frequent as those with potential temporal changes $\left(\right.$ Both $\left._{\text {temporal }}\right)$

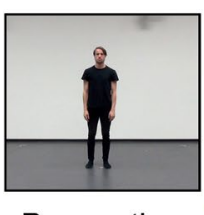

Preparation
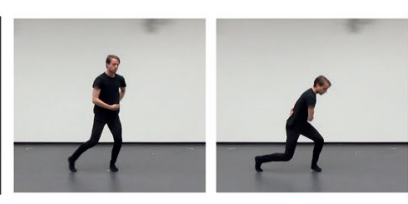

Movement unit 1
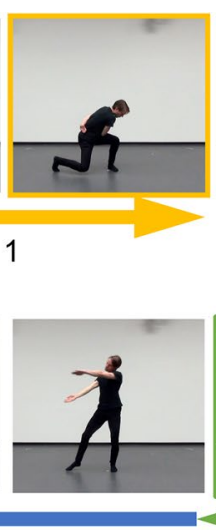

Time

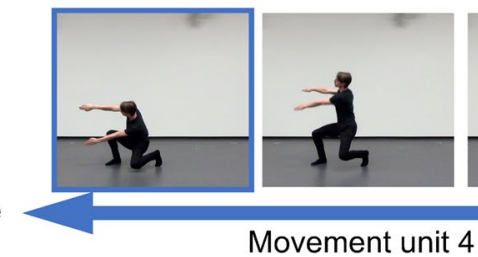

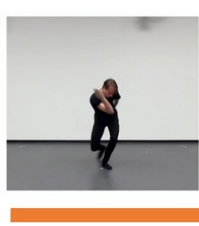

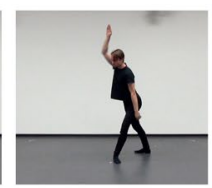

Movement unit 2
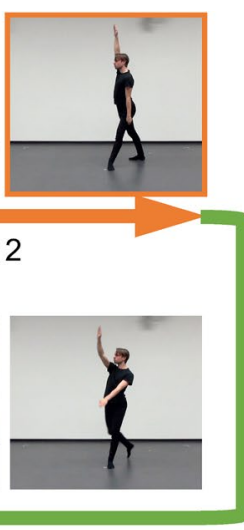

Movement unit 3
Fig. 1 Illustration of a whole-body movement sequence. Movement sequences started at the center of the recording region with a relaxed standing pose, followed by four linked movement units, i.e., the end- ing pose of the first unit was the starting pose of the second one, and so on. Movements were all without interpretable external goals and action semantics (see Online Resource 1-3 for sample video clips) clear starting point and ending point where the velocity was zero. Moreover, to better resemble the continuous nature of real-world human movements, the ending pose of the first unit was linked with the starting pose of the second one, and so on, to create a relatively continuous trajectory. In other words, each unit of a sequence had its own velocity profile, movement pattern, and use of body parts, but formed a continuous trajectory when aligned in a specific order (see Fig. 1). Movement sequences were performed by two professional dancers (half by a female dancer and the other half by a male dancer, both in fitted black clothing) at the center of a $3 \mathrm{~m} \times 3 \mathrm{~m}$ recording region with a maximum radius of approximate one step outward from the center point. In addition, movements were all without interpretable external goals and action semantics to diminish the influence from long-term semantic memory.

\section{Footnote 3 (continued)}

starting point and ending point can be identified and the manipulation of movement speed can be precisely implemented.

\section{Rhythm design}

Each movement sequence was performed in four different rhythms $(3212,2132,1223$, and 2321). All were eight beats in a 4/4 musical meter and paced at a tempo of 90 beats per minute, yielding a sequence length of about $6 \mathrm{~s}$ after including one additional beat for preparation. Each rhythm was composed of four temporal durations, one 1-beat duration (1), two 2-beat durations (2), and one 3-beat duration (3), corresponding to four movement units of a sequence. When the same trajectory was performed, a shorter duration also implied a higher speed. In addition, the four rhythms used in the current study were all metric complex rhythms (i.e., integer-ratio rhythms without regular temporal accents aligned with the beat), which were shown to be more difficult to induce beat perception than metric simple rhythms, such as 3122 or 1313 (Grahn 2012). Metric complex rhythms were used to avoid beat-based encoding. Note that positions of the shortest (1-beat) and the longest (3-beat) durations were balanced to take potential salience effects into account; total duration difference (by unit) between each of the two 
rhythms were the same with the smallest duration difference (i.e., one beat or $667 \mathrm{~ms}$ ) much longer than the temporal resolution of the visual modality $(\sim 100 \mathrm{~ms})$.

\section{Video recording, post-editing, and display}

Movement recordings were made with a digital video camera recorder (Sony HDR-CX430V) at 50 frames per second against a white background and a gray floor. The dancers performed each movement sequence in four different rhythms for at least two times with a metronome to ensure the consistency of trajectory and the precision of rhythm across different recordings. Videos were then edited on a frame basis using the software iMovie (Apple, Inc.) and presented silently (i.e., without the sound of metronome) to participants at $1600 \times 900$ pixels on a 24 -inch LCD screen (Dell U2412M) with a viewing distance of $\sim 50 \mathrm{~cm}$. The experimental flow and data analysis were programmed in Python, and stimuli presentation was implemented with the PsychoPy software package (Peirce 2007, 2008).

\section{Stimulus validation}

As mentioned previously, we intended to use a stimulus set of which the processing demand of spatial information was low to better probe the effect of task-driven attention on spatial information as opposed to the side effect (i.e., co-selection) resulting from temporal processing. The characteristic of the stimulus set had been validated by one of our previous studies ( $N=26$, unpublished data) wherein participants performed a similar change detection task as the one used in the current study. Results revealed that performance (measured by proportion correct) of detecting temporal changes of a four-unit sequence was about $70 \%$, while performance of detecting spatial changes was nearly perfect when both types of information were task-relevant.

\section{Procedure}

\section{Incidental encoding phase}

In the incidental encoding phase, participants performed a change detection task on whole-body movement sequences with two foci of attention: (1) Temporal-only and (2) Both. In the Temporal-only condition, they were instructed to attend to the movement speed to detect temporal changes; in the Both condition, they were instructed to pay additional attention to the movement path as there would be spatial changes as well. These two experimental conditions were performed in two separate sessions with an order counterbalanced between participants. The words path and speed (instead of trajectory and rhythm) were used in verbal instructions together with simplified animations illustrating these two types of differences (i.e., a square moving along a straight line or a curved line on a computer screen to illustrate a difference in "path", and a square moving along a straight line with a low or a high speed to illustrate a difference in "speed") to make the concepts more understandable to participants.

In the Temporal-only condition, the sample sequence and the test sequence were either the same (i.e., with the same trajectory and the same rhythm) or different in rhythm (i.e., same in trajectory). For example, the sequence A_3212 (with trajectory A and rhythm 3212) would form a same trial with the sequence A_3212 and a different trial with the sequence A_2132 (with trajectory A and rhythm 2132). As there were 4 pre-defined rhythms used in the current study, 6 types of temporal contrast (irrespective of order) can be formed for different trials. Note that for 2-unit sequences (see below), temporal contrast of 23 vs. 32 was not included due to less salience (i.e., more difficult to discriminate). In the Both condition, the sample sequence and the test sequence could be the same, or different in either rhythm (i.e., same in trajectory) or trajectory (i.e., same in rhythm), such as A_3212 vs. B_3212 (sequences with different trajectories $A$ and $B$, but the same rhythm 3212). Three sample video clips (A_2132, A_3212, B_3212) are provided in Online Resource 1-3.

The sequence length was also manipulated (as being 2 or 4 units) to examine whether the memory load might affect memory performance, and if yes, as predicted by the welldocumented set-size effect of working memory (i.e., short sequences are memorized better than long sequences) and illustrated by one of our previous studies (unpublished data), how this factor might influence the processing strategy (e.g., the allocation of attentional resources) when observing spatial and/or temporal information of whole-body movement sequences.

Each trial began with a 1-s fixation cross (+) followed by a sample sequence of 2 or 4 units. To control for the starting pose, a 2-unit sequence was defined as the first-half of a 4-unit sequence (i.e., unit $1+$ unit 2). A mask was presented for $0.5 \mathrm{~s}$ after the offset of the sample sequence. Following the mask, another 1-s fixation cross was presented before the display of the test sequence (in the same length as the sample sequence, i.e., 2 or 4 units), yielding a retention interval of $1.5 \mathrm{~s}$. After the offset of the test sequence, a mask for $0.5 \mathrm{~s}$ was presented, followed by a question "Are video 1 and video 2 the same?" Participants then made a yes/no judgment by keystroke on a standard computer keyboard ("F" key and "J" key, respectively, marked in red) (Fig. 2a). Before the start of each session, participants completed 8 practice trials with movement sequences not used in the formal experiment. In practice trials, participants were allowed to replay the sample sequence before making a judgment and received feedback (as shown by the word correct or 
a

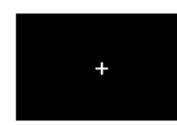

$1 \mathrm{~s}$

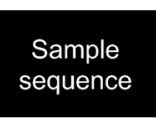

$2.7 s-6 s$

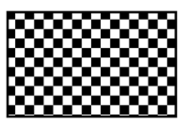

$0.5 \mathrm{~s}$

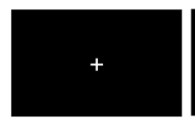

$1 \mathrm{~s}$

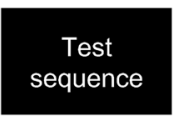

$2.7 s-6 s$

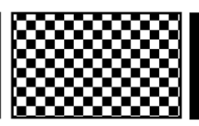

$0.5 \mathrm{~s}$
Are video 1 and video 2 the same?

Response (Yes/No) b

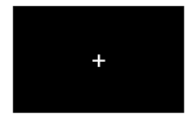

$1 \mathrm{~s}$

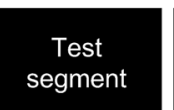

$0.7 s-3.4 s$
Have you seen this

movement before?

Response (Yes/No)
Fig. 2 Trial structure of the change detection task (same/different judgment) used in the incidental encoding phase (a) and the recognition task (old/new judgment) used in the surprise memory test phase (b). In the encoding phase, a sample sequence and a test sequence with the same length of 2 or 4 units were displayed sequentially with a delay of $1.5 \mathrm{~s}$ in between (including a mask for $0.5 \mathrm{~s}$ and a 1-s fixation cross before the display of the test sequence). Participants were required to detect changes in either temporal domain ("Temporalonly" condition) or in both spatial and temporal domains ("Both" condition). In the test phase, movement segments of 1 or 2 unit(s) extracted from observed or new sequences were used as test stimuli. Participants were required to make judgments on whether they have seen the movement before

was to create a natural segmentation to lower participants' expectation that the coming task might have a relation with the previous one. After the break, participants performed a surprise movement recognition task, in which movement segments (see below) extracted from 30 sequences observed in the encoding phase as well as 8 new sequences (designed and recorded under the same rules and condition as the old sequences) were used as test stimuli. It would be ideal if a balanced design could have been used, in which the number of old trials was equal to the number of new trials. However, given the stimulus pool we had (i.e., the one created for a previous study, see Footnote 2) and the difficulty of recording additional movement sequences, especially when low-level visual differences across different recordings were considered, we decided to accommodate our experimental design to the resources we had.

During the test, each movement unit of a sequence was displayed once (and only once) as either a 1-unit segment or as part of a 2 -unit segment (i.e., the first half, unit $1+$ unit 2 , or the second half, unit $3+$ unit 4 , of the original 4-unit sequence), which was randomly decided for each participant. As movement units were equally divided into these two types of segments, the number of 1-unit trials was twice as many as the number of 2-unit trials (see Table 1). We manipulated the segment length to test if 2-unit segments were recognized better than 1-unit segments. If the movement recognition was mediated by unit-based retrieval, the probability of "encountering" a remembered unit should be higher when observing 2-unit than 1-unit segments, leading to a better performance. Even if the recognition relied on sequence-based representations, we predicted that longer segments, which were 
supposed to provide additional contextual or ensemble information, would also be easier to recognize.

In addition, test segments varied in movement speed as did original sequences. Rhythms (or temporal durations) that accompanied test segments were the rhythms that accompanied the original 4-unit sequences in the same trials of the change detection task. Therefore, the distribution of temporal durations across all segments reflected the original design of the rhythms (see "Rhythm design"); namely, the number of 2-beat units was twice as many as the number of 1-beat or 3-beat units.

Each trial began with a 1-s fixation cross $(+)$, followed by a test segment and then a question "Have you seen this movement before?" Participants were asked to make a yes/ no response based on the "movement" itself, irrespective of the speed (Fig. 2b). In total, 114 trials were completed (with 90 old segments from 30 old sequences and 24 new segments from 8 new sequences) (see Table 1). No practice trials were provided in this phase. The recognition task took about 8 min to complete.

\section{Data analyses}

Based on the signal detection theory (SDT) (Green and Swets 1966; Macmillan and Creelman 1991), we used the proportion of correct responses, defined as an average of hit rate (correctly responding "same" on same trials or "old" on old trials) and correct-rejection rate (correctly responding "different" on different trials or "new" on new trials), as well as the sensitivity measures $d^{\prime}$ and $A^{\prime}$ (Stanislaw and Todorov 1999), where response biases are taken into account, to evaluate participants' performance in the change detection task and the recognition task.

As we intentionally used a stimulus set of which participants' sensitivity to spatial changes was much higher than that to temporal changes, this sensitivity difference should be considered when calculating domain-specific performance measures under the Both condition (i.e., when two feature dimensions were involved in the decision space). Specifically, an overall hit rate obtained from the same trials reflected an evaluation of evidence distributed across spatial and temporal dimensions. Given the high discriminability of spatial information in the current study, the overall hit rate may highly underestimate participants' performance in the spatial domain. To solve this problem, we used an adapted approach inspired by Luan et al. (2011), combining the SDT analysis with a two-cue fast-and-frugal tree (FFT) to calculate domain-specific hit rates and false alarm rates (incorrectly responding "same" on different trials, i.e., complementary to correct-rejection rates) when both spatial and temporal information were task-relevant.

In tasks where a binary decision needs to be made with $m$ decision cues available, an FFT is defined as a decision tree that has $m+1$ exits, with one exit for each of the first $m-1$ cues and two exits for the last cue to ensure that a final decision will be made (Luan et al. 2011). In the Both condition of the change detection task, there were two decision cues embedded in the question "Are video 1 and video 2 the same?", namely "Do they have the same trajectory?" and "Do they have the same rhythm?" A two-cue FFT can thus be constructed based on these two decision cues and a pre-defined $2 \mathrm{~s}$ (signal)-1n (noise) exit rule, meaning that participants were required to make a "same" judgment when both trajectory and rhythm were the same (2s), but a "different" judgment when either trajectory or rhythm was different (1n) (see Fig. 3). This decision rule was made explicit and clearly emphasized in verbal instructions. In addition, as the discriminability of spatial information (trajectory) was assumed to be higher than that of temporal information (rhythm), participants were expected to make judgments based on the spatial cue first. But since there were only two cues in this FFT, the cue order would not influence the sensitivity of the FFT.

The overall hit rate calculated from the same trials $\left(P(\text { Hit })_{\text {same }}\right)$ and the false alarm rates calculated from the different trials with spatial $\left(P(\mathrm{FA})_{\text {spatial }}\right)$ or temporal $\left(P(\mathrm{FA})_{\text {temporal }}\right)$ changes can be expressed as the following:

$$
\begin{aligned}
& P(\mathrm{Hit})_{\mathrm{same}}=P\left[\left(x_{\mathrm{ss}}>x_{\mathrm{cs}}\right) \cap\left(x_{\mathrm{st}}>x_{\mathrm{ct}}\right)\right]=\mathrm{Hit}_{\mathrm{s}} \times \mathrm{Hit}_{\mathrm{t}} \\
& P(\mathrm{FA})_{\text {spatial }}=P\left[\left(x_{\mathrm{ns}}>x_{\mathrm{cs}}\right) \cap\left(x_{\mathrm{st}}>x_{\mathrm{ct}}\right)\right]=\mathrm{FA}_{\mathrm{s}} \times \mathrm{Hit}_{\mathrm{t}} \\
& P(\mathrm{FA})_{\text {temporal }}=P\left[\left(x_{\mathrm{ss}}>x_{\mathrm{cs}}\right) \cap\left(x_{\mathrm{nt}}>x_{\mathrm{ct}}\right)\right]=\mathrm{Hit}_{\mathrm{s}} \times \mathrm{FA}_{\mathrm{t}}
\end{aligned}
$$

where $x_{\mathrm{ss}}$ and $x_{\mathrm{ns}}$ are decision variables in the spatial domain when drawn from signal or noise category; $x_{\mathrm{st}}$ and $x_{\mathrm{nt}}$ are decision variables in the temporal domain when drawn from signal or noise category; $x_{\mathrm{cs}}$ and $x_{\mathrm{ct}}$ are decision criteria in the spatial and temporal domains, respectively. The hit rates and false alarm rates of the two decision cues are denoted as $\mathrm{Hit}_{\mathrm{s}}, \mathrm{FA}_{\mathrm{s}}$ (spatial domain), and $\mathrm{Hit}_{\mathrm{t}}, \mathrm{FA}_{\mathrm{t}}$ (temporal domain). In addition, since incorrect judgments in both same and different trials mainly resulted from the same inaccurate working memory representation of the spatial and/or temporal information of the sample sequence, we made an additional assumption that participants' miss rates (incorrectly responding "different" on same trials, i.e., complementary to hit rates), denoted as "Miss", were proportional to their false alarm rates in the respective domains:

$\operatorname{Miss}_{\mathrm{s}}: \operatorname{Miss}_{\mathrm{t}}=\left(1-\mathrm{Hit}_{\mathrm{s}}\right):\left(1-\mathrm{Hit}_{\mathrm{t}}\right)=\mathrm{FA}_{\mathrm{s}}: \mathrm{FA}_{\mathrm{t}}$

If $\mathrm{FA}_{\mathrm{s}}=\mathrm{FA}_{\mathrm{t}}=\mathrm{O}, \mathrm{Hit}_{\mathrm{s}}$ and $\mathrm{Hit}_{\mathrm{t}}$ would be unsolvable. Under this condition, if $P(\mathrm{Hit})_{\text {same }}=1$, it was assumed that $\mathrm{Hit}_{\mathrm{s}}=\mathrm{Hit}_{\mathrm{t}}=1$ (i.e., perfect discrimination); if $P(\mathrm{Hit})_{\mathrm{same}}<1$, 


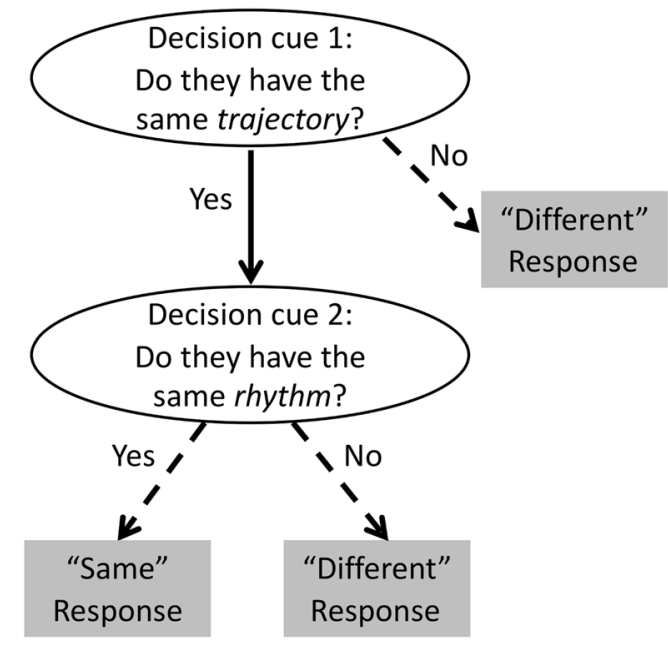

Fig. 3 A two-cue fast-and-frugal tree (FFT) and two sets of signal and noise probability density distributions according to the signal detection theory (SDT). The discriminability of spatial information (trajectory) is assumed to be higher than that of temporal information (rhythm), and thus the spatial cue is more likely to be used as the first decision cue. Decision criteria are placed where the noise and signal

false alarm rates of a similar within-subjects category would be used as an approximation. For example, the ratio of $\mathrm{FA}_{\mathrm{s}}$ to $\mathrm{FA}_{\mathrm{t}}$ of 4-unit sequences would be used as an approximation for that of 2-unit sequences. If no within-subjects data were available, a group average would be used instead. By solving Eqs. (1)-(4), hit rates and false alarm rates for spatial and temporal information (i.e., $\mathrm{Hit}_{\mathrm{s}}, \mathrm{FA}_{\mathrm{s}}$, and $\mathrm{Hit}_{\mathrm{t}}, \mathrm{FA}_{\mathrm{t}}$ ) as well as the proportion correct and the sensitivity measures $d^{\prime}$ and $A^{\prime}$ of respective feature dimensions can be calculated.

We set the statistical threshold of Type I error at $\alpha=$ .05 and reported Cohen's $d$ and partial eta squared $\left(\eta_{\mathrm{p}}^{2}\right)$ to indicate effect size. Post hoc analyses were conducted using Bonferroni correction.

\section{Results}

\section{Incidental encoding phase}

We first checked if participants' discrimination performance for spatial changes was high as expected. The results revealed a proportion correct of $98.1 \%(\mathrm{SD}=3.94 \%)$ for 2-unit sequences and $96.1 \%(\mathrm{SD}=7.06 \%)$ for 4 -unit sequences when both spatial and temporal information were task-relevant. The close-to-ceiling performance indicates that the processing demand of spatial information was low and thus the additional processing demand of spatial information might not decrease the amount of attentional resources that were available for temporal processing. To verify this prediction and to check if the order of performing
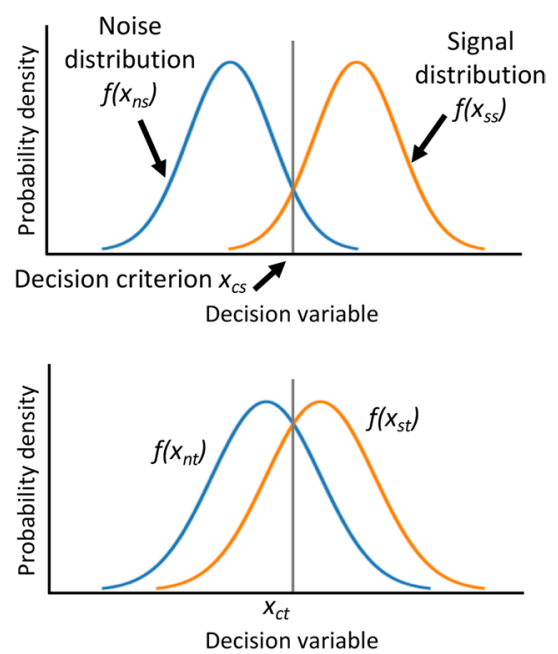

distributions intersect for simplicity, while each cue may have its own decision criterion, which is not necessarily to be unbiased. $f\left(x_{s s}\right)$ and $f\left(x_{n s}\right)$ are signal and noise distributions in the spatial domain; $f\left(x_{s t}\right)$ and $f\left(x_{n t}\right)$ are signal and noise distributions in the temporal domain; $x_{c s}$ and $x_{c t}$ are decision criteria in the spatial and temporal domains, respectively. Dashed arrows indicate that a final decision is made

the two experimental sessions (i.e., starting with the Temporal-only condition, followed by the Both condition, or the other way around) might also affect performance, we conducted a 2 (focus of attention: temporal-only, both) $\times 2$ (sequence length: 2 -unit, 4-unit) $\times 2$ (order: temporalonly + both, both + temporal-only) mixed analysis of variance (ANOVA) on temporal performance (measured by proportion correct), with "focus of attention" and "sequence length" as the within-subjects factors and "order" as the between-subjects factor.

Descriptive statistics of each performance measure under respective experimental conditions are summarized in Table 2. The ANOVA yielded a significant two-way interaction between focus of attention and order, $F(1,36)=4.84$, $p=0.034, \eta_{\mathrm{p}}{ }^{2}=0.12$. Pairwise comparisons (collapsing sequence length) indicated that participants' sensitivity to temporal changes remained the same irrespective of whether they attended to the temporal information only $(M=75.0 \%$, $\mathrm{SD}=12.3 \%$ ) or additionally also to the spatial information $(M=73.0 \%, \mathrm{SD}=8.20 \%)$ if they started with the Both condition, $t(19)=-0.88, p=0.390, d=-0.20$. Surprisingly, for participants who started with the Temporal-only condition, their performance improved in the second session when additional spatial information was required to be processed $(M=78.1 \%, \mathrm{SD}=6.74 \%$ in the Both condition; $M=74.1 \%$, $\mathrm{SD}=7.36 \%$ in the Temporal-only condition), $t(17)=2.53$, $p=0.021, d=0.60$, illustrating a potential learning effect (Fig. 4a).

One may suspect that the performance improvement might alternatively indicate that actively attending to 
Table 2 Performance of the change detection task in the incidental encoding phase by order, sequence length, and focus of attention

\begin{tabular}{|c|c|c|c|c|}
\hline & \multicolumn{2}{|c|}{$\begin{array}{l}\text { Order: temporal- } \\
\text { only + both }\end{array}$} & \multicolumn{2}{|c|}{$\begin{array}{l}\text { Order: both + temporal- } \\
\text { only }\end{array}$} \\
\hline & 2-unit & 4-unit & 2-unit & 4-unit \\
\hline \multicolumn{5}{|c|}{ Temporal-only condition } \\
\hline$P(\text { Hit })_{\text {same }}$ & $.83(.12)$ & $.64(.19)$ & $.82(.16)$ & $.70(.21)$ \\
\hline$P(\mathrm{FA})_{\text {temporal }}$ & $.22(.13)$ & $.29(.15)$ & $.23(.18)$ & $.28(.18)$ \\
\hline $\begin{array}{l}\text { Proportion cor- } \\
\text { rect }\end{array}$ & $.81(.08)$ & $.68(.10)$ & $.79(.12)$ & $.71(.16)$ \\
\hline$d^{\prime}$ & $1.87(0.62)$ & $1.03(0.59)$ & $1.84(0.85)$ & $1.27(1.01)$ \\
\hline$A^{\prime}$ & $.88(.06)$ & $.75(.12)$ & $.86(.11)$ & $.78(.18)$ \\
\hline \multicolumn{5}{|l|}{ Both condition } \\
\hline$P(\text { Hit })_{\text {same }}$ & $.83(.14)$ & $.73(.13)$ & $.82(.14)$ & $.69(.16)$ \\
\hline $\mathrm{Hit}_{\mathrm{s}}$ & $.98(.04)$ & $.95(.08)$ & $.99(.02)$ & $.96(.08)$ \\
\hline $\mathrm{Hit}_{\mathrm{t}}$ & $.84(.14)$ & $.77(.12)$ & $.83(.14)$ & $.71(.15)$ \\
\hline$P(\mathrm{FA})_{\text {spatial }}$ & $.03(.06)$ & $.03(.05)$ & $.02(.03)$ & $.03(.05)$ \\
\hline $\mathrm{FA}_{\mathrm{s}}$ & $.03(.07)$ & $.04(.06)$ & $.02(.04)$ & $.03(.07)$ \\
\hline$P(\mathrm{FA})_{\text {temporal }}$ & $.19(.13)$ & $.29(.17)$ & $.28(.21)$ & $.33(.20)$ \\
\hline $\mathrm{FA}_{\mathrm{t}}$ & $.19(.13)$ & $.30(.16)$ & $.29(.21)$ & $.34(.20)$ \\
\hline \multicolumn{5}{|c|}{ Proportion correct } \\
\hline Spatial & $.97(.05)$ & $.96(.07)$ & $.99(.02)$ & $.96(.07)$ \\
\hline Temporal & $.83(.09)$ & $.74(.08)$ & $.77(.11)$ & $.69(.07)$ \\
\hline \multicolumn{5}{|l|}{$d^{\prime}$} \\
\hline Spatial & $3.49(0.58)$ & $3.29(0.75)$ & $3.67(0.25)$ & $3.40(0.74)$ \\
\hline Temporal & $2.10(0.81)$ & $1.38(0.52)$ & $1.75(0.81)$ & $1.13(0.46)$ \\
\hline \multicolumn{5}{|l|}{$A^{\prime}$} \\
\hline Spatial & $.99(.03)$ & $.97(.04)$ & $.99(.01)$ & $.98(.04)$ \\
\hline Temporal & $.89(.07)$ & $.82(.07)$ & $.85(.11)$ & $.78(.07)$ \\
\hline
\end{tabular}

Numbers in the parentheses are standard deviations. $P(\mathrm{Hit})_{\text {same }}$ is the hit rate calculated from the same trials; $P(\mathrm{FA})_{\text {spatial }}$ and $P(\mathrm{FA})_{\text {temporal }}$ are false alarm rates calculated from the different trials with spatial and temporal changes, respectively. $\mathrm{Hit}_{\mathrm{s}}$ and $\mathrm{Hit}_{\mathrm{t}}$ are domain-specific hit rates for spatial and temporal information; $\mathrm{FA}_{\mathrm{s}}$ and $\mathrm{FA}_{\mathrm{t}}$ are domain-specific false alarm rates for spatial and temporal information. Proportion correct, $d^{\prime}$, and $A^{\prime}$ for spatial and temporal information in the Both condition were calculated based on the domain-specific hit rates $\left(\mathrm{Hit}_{\mathrm{s}}, \mathrm{Hit}_{\mathrm{t}}\right)$ and false alarm rates $\left(\mathrm{FA}_{\mathrm{s}}, \mathrm{FA}_{\mathrm{t}}\right)$

spatial information was beneficial for detecting temporal changes. However, if that was the case, participants who started with the Both condition should also have performed better in this specific condition, namely that we should have observed a performance decline in the Temporalonly condition in this group, but we did not. Moreover, when leaving out potential order effects by comparing the first-session performance of the two order groups, we did not find any significant difference between the Both and the Temporal-only conditions, $t(36)=-1.05, p=0.299$, $d=-0.34$ for short sequences; $t(30.6)=0.43, p=0.671$, $d=0.14$ for long sequences, indicating that the active attention to spatial information did not benefit temporal discrimination.
Across the two order conditions, as the temporal performance was far from ceiling (e.g., $M=79.8 \%, \mathrm{SD}=10.4 \%$ for 2-unit sequences; $M=69.3 \%, \mathrm{SD}=13.0 \%$ for 4 -unit sequences in the Temporal-only condition), it is reasonable to assume that any re-allocation of attentional resources from temporal to spatial processing would have impaired temporal performance, as no spare capacity was available. The results therefore demonstrated that by adding a low demand on spatial processing, we successfully manipulated participants' focus of attention (through task requirements) while keeping the redistribution of attentional resources at a minimal level. The analysis conducted on the sensitivity measures $d^{\prime}$ and $A^{\prime}$ also led to the same conclusion. The analysis on $d^{\prime}$ again yielded an interaction effect between focus of attention and order, $F(1,36)=4.78, p=0.035, \eta_{\mathrm{p}}{ }^{2}=0.12$. Participants who started with the Temporal-only condition performed better in the Both condition, $t(17)=2.69, p=0.015, d=0.63$, while no such difference was shown for participants who started with the Both condition, $t(19)=-0.75, p=0.461, d=-0.17$. The analysis conducted on $A^{\prime}$ did not reveal a significant effect of focus of attention for both order groups, $F(1,36)=1.92$, $p=0.175, \eta_{\mathrm{p}}^{2}=0.05$.

The main effect for sequence length was significant, proportion correct: $F(1,36)=49.5, p<0.001, \eta_{\mathrm{p}}{ }^{2}=0.58 ; d^{\prime}$ : $F(1,36)=51.8, p<0.001, \eta_{\mathrm{p}}{ }^{2}=0.59 ; A^{\prime}: F(1,36)=31.3$, $p<0.001, \eta_{\mathrm{p}}^{2}=0.47 ; 2$-unit sequences were discriminated better than 4-unit sequences, illustrating the set-size effect of working memory. No interaction effect was found between focus of attention and sequence length, proportion correct: $F(1,36)=0.73, p=0.398, \eta_{\mathrm{p}}{ }^{2}=0.02 ; d^{\prime}$ : $F(1,36)=0.04, p=0.839, \eta_{\mathrm{p}}{ }^{2}=0.001 ; A^{\prime}: F(1,36)=1.89$, $p=0.178, \eta_{\mathrm{p}}{ }^{2}=0.05$, indicating that an increase of cognitive load did not affect the processing strategy or the way attention deployed during observation. No other effects were significant.

\section{Surprise memory test phase}

Recognition performance (measured by proportion correct after considering the false alarm rate obtained from the new trials) for movements previously displayed in the Temporal-only condition was $71.3 \%(\mathrm{SD}=8.60 \%)$ for 1 -unit segments and $81.3 \%(\mathrm{SD}=10.5 \%)$ for 2-unit segments; both were higher than the chance level of $50 \%$, indicating that the task-irrelevant spatial information was not only perceived but also encoded in memory. In addition, to examine whether the task-driven attention provided extra benefits in terms of the long-term retention of the attended information, we conducted a 2 (focus of attention: temporal-only, both) $\times 2$ (segment length: 1-unit, 2-unit) $\times 2$ (order: temporal-only + both, both + temporal-only) mixed ANOVA on recognition performance, with "focus of attention" and "segment length" as the within-subjects factors and "order" 


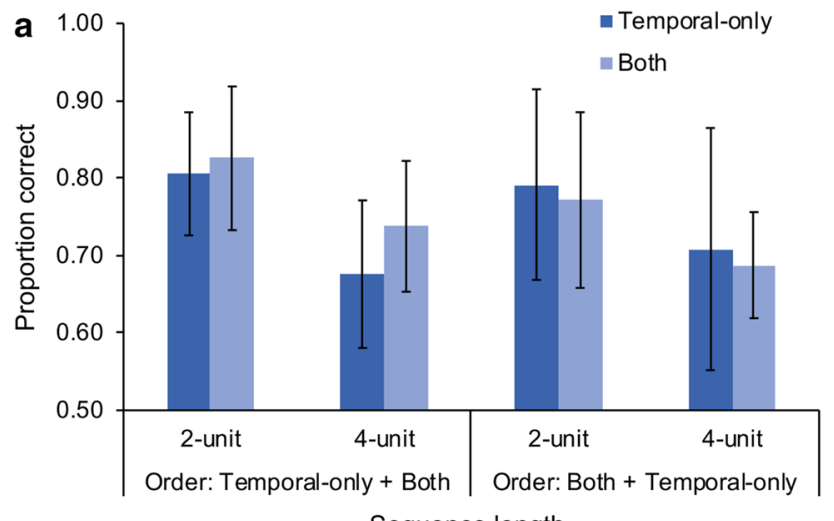

Sequence length

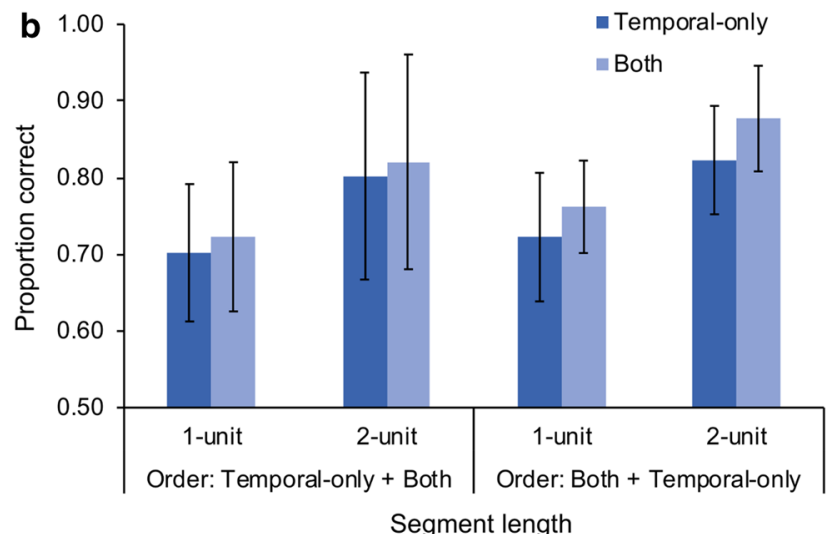

Fig. 4 Temporal performance of the change detection task in the incidental encoding phase (a) and recognition performance in the memory test phase (spatial fillers were excluded) (b) Error bars indicate one standard deviation of the mean

as the between-subjects factor. Spatial fillers were excluded from analyses (but see Table 3 for a summary of descriptive statistics). We added "order" as an additional factor to test whether participants might process the observed information in different ways depending on which session they performed at first. Specifically, participants who attended to temporal changes in the first session might bias their attention towards temporal information in the second session (i.e., Both condition), resulting in a worse recognition performance in the test phase. On the contrary, participants who attended to both spatial and temporal changes in the first session might process spatial information to a deeper extent even when it was task-irrelevant in the second session (i.e., Temporal-only condition), leading to a better recognition performance.

The results showed a significant main effect for focus of attention, $F(1,36)=8.75, p=0.005, \eta_{\mathrm{p}}{ }^{2}=0.20$; movements whose trajectories were intentionally attended to in the Both condition $(M=79.6 \%, 95 \%$ CI $[76.9 \%, 82.2 \%])$ were recognized better than those in the Temporal-only condition $(M=76.2 \%, 95 \%$ CI $[73.5 \%, 79.0 \%])$. The finding indicates a modulation from attention on incidental memory encoding (Fig. 4b). The main effect for segment length was also significant, $F(1,36)=53.6, p<0.001, \eta_{\mathrm{p}}^{2}=0.60 ; 2$-unit segments $(M=83.1 \%, 95 \%$ CI $[79.9 \%, 86.3 \%])$ were recognized better than 1-unit segments $(M=72.7 \%, 95 \%$ CI $[70.3 \%, 75.2 \%])$, consistent with our prediction that long sequences were recognized better than short sequences. The same conclusion can also be drawn when the analysis was conducted on $d^{\prime}$ and $A^{\prime}$, respectively. Movement segments previously displayed in the Both condition were recognized better than those displayed in the Temporal-only condition, $d^{\prime}: F(1,36)=8.25, p=0.007, \eta_{\mathrm{p}}{ }^{2}=0.19 ; A^{\prime}: F(1,36)=7.06$, $p=0.012, \eta_{\mathrm{p}}^{2}=0.16$, and 2-unit segments were recognized better than 1-unit segments, $d^{\prime}: F(1,36)=50.0, p<0.001$, $\eta_{\mathrm{p}}{ }^{2}=0.58 ; A^{\prime}: F(1,36)=35.5, p<0.001, \eta_{\mathrm{p}}^{2}=0.50$.
The main effect for order was not significant, proportion correct: $F(1,36)=2.01, p=0.165, \eta_{\mathrm{p}}{ }^{2}=0.05 ; d^{\prime}$ : $F(1,36)=2.40, p=0.130, \eta_{\mathrm{p}}^{2}=0.06 ; A^{\prime} F(1,36)=2.93$, $p=0.096, \eta_{\mathrm{p}}^{2}=0.08$, indicating that the performance order did not influence the extent to which the spatial information

Table 3 Performance of the recognition task in the surprise memory test phase by order, segment length, and probe condition

\begin{tabular}{|c|c|c|c|c|}
\hline & \multicolumn{2}{|c|}{$\begin{array}{l}\text { Order: temporal- } \\
\text { only + both }\end{array}$} & \multicolumn{2}{|c|}{$\begin{array}{l}\text { Order: both + temporal- } \\
\text { only }\end{array}$} \\
\hline & 1-unit & 2-unit & 1-unit & 2-unit \\
\hline \multicolumn{5}{|l|}{ Hits } \\
\hline Temporal-only & $.66(.16)$ & $.75(.17)$ & $.65(.17)$ & $.76(.15)$ \\
\hline Both $_{\text {temporal }}$ & $.71(.15)$ & $.79(.14)$ & $.73(.12)$ & $.87(.12)$ \\
\hline Both $_{\text {filler }}$ & $.75(.22)$ & $.94(.16)$ & $.84(.16)$ & $.92(.10)$ \\
\hline \multicolumn{5}{|l|}{ Correct rejection } \\
\hline New & $.74(.12)$ & $.85(.18)$ & $.79(.14)$ & $.89(.10)$ \\
\hline \multicolumn{5}{|c|}{ Proportion correct } \\
\hline Temporal-only & $.70(.09)$ & $.80(.14)$ & $.72(.08)$ & $.82(.07)$ \\
\hline Both $_{\text {temporal }}$ & $.72(.10)$ & $.82(.14)$ & $.76(.06)$ & $.88(.07)$ \\
\hline $\begin{array}{l}\text { Both }_{\text {filler }} \\
d^{\prime}\end{array}$ & $.74(.12)$ & $.89(.16)$ & $.82(.09)$ & $.91(.07)$ \\
\hline Temporal-only & $1.16(0.55)$ & $1.83(0.89)$ & $1.33(0.54)$ & $1.94(0.49)$ \\
\hline Both $_{\text {temporal }}$ & $1.28(0.62)$ & $1.93(0.91)$ & $1.56(0.42)$ & $2.33(0.52)$ \\
\hline $\begin{array}{l}\text { Both }_{\text {filler }} \\
A^{\prime}\end{array}$ & $1.49(0.81)$ & $2.27(0.92)$ & $1.98(0.66)$ & $2.36(0.40)$ \\
\hline Temporal-only & $.78(.10)$ & $.86(.13)$ & $.81(.08)$ & $.90(.05)$ \\
\hline Both $_{\text {temporal }}$ & $.80(.10)$ & $.87(.14)$ & $.85(.05)$ & $.93(.05)$ \\
\hline Both $_{\text {filler }}$ & $.82(.11)$ & $.92(.16)$ & $.89(.07)$ & $.95(.04)$ \\
\hline
\end{tabular}

Numbers in the parentheses are standard deviations. Performance

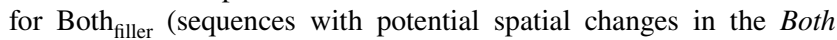
condition, i.e., spatial fillers) is not directly comparable with perfor-

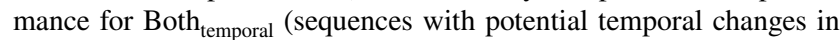
the Both condition), as Both filler $_{\text {were displayed twice as frequent as }}$ Both $_{\text {temporal }}$ in the incidental encoding phase 
was processed. Nevertheless, descriptive statistics showed a tendency that participants who started with the Temporalonly condition did not recognize as many movement segments from the Both condition (measured by hit rate) as those who started with the Both condition (see Table 3). The finding suggests that participants who attended to temporal changes first might have biased their attention more towards temporal information in the Both condition than those who started with the Both condition, despite the fact that no significant difference was shown in the spatial discrimination performance between these two order groups, $t(36)=-0.64$, $p=0.526, d=-0.21$. However, participants who attended to the spatial information in the first session did not show a similar spatial bias in the Temporal-only condition. Their recognition performance for movements from the Temporal-only condition was comparable with that of those who started with the Temporal-only condition (see Table 3). This difference in processing strategy might result from the difference in sensitivity between spatial and temporal processing. No other effects were significant.

\section{Discussion}

In this study, we used complex whole-body movement sequences to investigate the extent to which the task-irrelevant spatial information (movement trajectory) is processed when only the temporal information (movement rhythm) is in focus. We found that the task-irrelevant spatial information was not only perceived, as predicted by the dependence of temporal processing on spatial information (Casasanto and Boroditsky 2008; Casasanto et al. 2010; Dormal and Pesenti 2013; Santiago et al. 2011; Starr and Brannon 2016), but also encoded in memory, consistent with previous studies showing that durable memory representations can be formed for unattended or task-irrelevant information (e.g., Hutmacher and Kuhbandner 2020; Kuhbandner et al. 2017). In addition, we examined whether attention that is actively directed to spatial information provides extra benefits when compared with unintended co-selection or attentional spreading resulting from temporal processing. The answer is yes. We found that movements whose trajectories were intentionally attended to during observation were recognized better in a later memory test than those that were not, indicating a modulation from attention on information processing and incidental memory encoding.

Note that the temporal performance in the incidental encoding phase was not impaired by additional demand from spatial processing, suggesting that a similar amount of cognitive resources were allocated to the temporal processing in the Both condition as in the Temporal-only condition and that no significant redistribution of attentional resources had occurred between these two experimental conditions.
Although there might be a potential difference in processing strategy depending on which session, Temporal-only or Both, was performed at first, the results indicate that all participants, irrespective of their performance order, tended to allocate a maximum possible amount of attentional resources to temporal processing across the Temporal-only and Both conditions. For participants who started with the Temporal-only condition, it was a bias towards temporal information in the Both condition (i.e., restricting the additional spatial processing required by the task to a minimum level); for participants who started with the Both condition, it was a selective attention to temporal processing in the Temporal-only condition (i.e., without a notable spatial bias). As indicated previously, this difference in processing strategy might result from the sensitivity difference between spatial and temporal processing. It is worth noting that the interaction effect we found between focus of attention and order in the change detection task was not reflected in the memory behavior observed in the recognition task. The finding may suggest that working memory representations and visual cues participants used for detecting changes between two sequentially displayed movement sequences might differ qualitatively from long-term memory representations and retrieval cues they used for recognizing movements.

Moreover, the finding that the additional demand from spatial processing did not impair temporal discrimination also implies that processing temporal information required or "co-occurred" with a certain level of spatial processing, and this level of processing was already sufficient to fulfill the task requirement in the spatial domain. Although the finding provides further evidence in support of the dependence of temporal processing on spatial information, it is so far unclear whether temporal information is fully integrated with the corresponding spatial information or it is partially dependent on it. Previous research proposes that different features of a movement (e.g., action type, duration) are bound together and stored as an integrated representation in working memory (Wood 2007, 2011), consistent with the view of "object-based storage" assuming an automatic integration of features into objects (Luck and Vogel 1997). Nevertheless, the current results showing that the spatial processing can be modulated independently from temporal processing by focus of attention imply that spatial and temporal information, albeit interdependent, might not be fully (and compulsorily) integrated.

If the change of attentional focus did not lead to resource redistribution between spatial and temporal processing, how did attention modulate incidental memory encoding of spatial information? One possibility is, although the same amount of cognitive resources might be allocated to temporal processing in both experimental conditions, different aspects of spatial information might be processed when different attentional foci were adopted. As suggested by 
Cavanagh (1991), passive motion processing is responsible for low-level motion detection, which is accomplished relatively automatically via parallel arrays of motion detectors, while active motion processing involves the use of attention to track selective features across time. The passive/ active distinction therefore implies that information that is extracted by passive motion processing might be fundamentally different from that extracted by active motion processing (Thornton et al. 2002). In the current study, for example, a low-resolution movement configuration might be processed when only temporal judgements were to be made, while active attention might be directed to the movement of specific body parts when additional spatial judgements were to be made. Although these two types of information may be equally beneficial for temporal processing, the latter may provide more cues for movement recognition in the test phase. In other words, spatial features that participants captured and used for the change detection (and the later recognition) task might depend on task requirements or, more specifically, focus of attention.

This explanation resonates with the distinction between attention as mental effort (or resource) and attention as selective processing (Chun and Turk-Browne 2007; Johnston and Dark 1986). Previous studies have shown that implicit learning, for example, can occur independently of attentional load (such as when a cognitive-demanding secondary task is introduced), but requires task-relevant stimuli to be selectively attended (Jiang and Chun 2001; Jiménez and Mendez 1999; Turk-Browne et al. 2005). Our finding also suggests that attention can enhance memory not necessarily through the deployment of additional resources, but through a selective process, in which features that are relevant to task performance are selected for processing. Further studies would be required to examine which types of information are most likely to be extracted under which types of attentional foci and task requirements.

Alternatively, it might be possible that the better recognition performance for movements previously displayed in the Both condition was due to a deeper level of information processing required by the task rather than a direct modulation from attention. Specifically, when performing a change detection task, participants need to store the observed information (sample sequence) temporarily in memory before it can be compared with the subsequent information (test sequence). That is, when spatial judgments were required, spatial information was actively stored in memory for a short period of time (retention interval) before the judgments were made. This additional processing may enhance the incidental memory encoding and thus increase the probability of successful retrieval. Although this explanation is plausible, we do not expect large effects from working memory. In one of our previous studies (unpublished data), participants' performance in a similar change detection task was close to ceiling even with a retention interval of $7 \mathrm{~s}$. Therefore, the working memory demand imposed by a retention interval of $1.5 \mathrm{~s}$ in the current study should be low and even neglectable. Moreover, as spatial fillers (movement sequences used in the trials with spatial changes) were excluded from analyses, the better recognition performance for movements displayed in the Both condition cannot be attributed to change-induced effects (such as attentional capture) in the spatial domain during the encoding phase.

Although our finding indicates that the task-irrelevant spatial information was not only perceived, but also encoded in memory as illustrated by the above chance recognition performance in the surprise memory test phase, one should note that the current finding is based on an experimental design of which the processing demand of spatial information was low (i.e., spatial discriminability was high) and the processing demand of temporal information was high (i.e., temporal discriminability was low). In other words, the task-irrelevant spatial information might have been processed to a lesser extent if the discriminability of temporal information has been increased or the salience (but not discriminability, see below) of spatial information has been decreased. According to the attentional spreading account of object-based attention, the processing of task-relevant and task-irrelevant information would be enhanced simultaneously by attentional focus if relevant and irrelevant information pertain to the same object (Chen 2003; Cosman and Vecera 2012; Richard et al. 2008). This would predict that an increase of temporal discriminability might decrease the corresponding processing of spatial information due to an overall decrease in attentional demand. Moreover, reducing the salience of task-irrelevant spatial information might also suppress its processing and lessen its chance of being retained in memory.

Note that the "salience" here refers to the characteristics of movements per se rather than the spatial discriminability between two movement sequences. Based on the current design, a decrease of spatial discriminability would be irrelevant to the Temporal-only condition and the co-processing of spatial information, as there would be only one same movement sequence (i.e., trajectory) displayed in each trial, either with or without a change in rhythm. A decrease of spatial discriminability, however, would increase the processing demand of spatial information in the Both condition, requiring more cognitive resources to be allocated to the spatial domain (i.e., less to the temporal domain when compared with the Temporal-only condition). This resource re-allocation is what we tried to avoid (or minimize), as it would make the comparison between the effect of "passive co-selection" and the effect of "active task-driven attention" less interesting, since one can easily attribute the extra benefits to the larger amount of cognitive resources allocated to the attended information in the latter condition. 
Furthermore, one should also note that human body and movement is a type of information that is not only complex, but also highly familiar and socially relevant to all human beings. The fact that most people are extremely efficient in biological movement perception and sensitive to body related changes (Blake and Shiffrar 2007; Peelen and Downing 2007) would imply that the task-irrelevant spatial information of human movements might be more difficult to suppress in general than that of other types of complex or dynamic stimuli.

In conclusion, this study provides evidence in support of a relatively automatic co-selection of task-irrelevant spatial information in temporal processing by using more ecologically-valid and complex movement stimuli. Although this finding might be well expected given the intrinsic spatiotemporal dependence in human movements, what we found more interesting is that the unintended coselection of task-irrelevant information did not prevent active attention from further enhancing its processing. In other words, the task-driven active attention provided additional benefits, expressed as a deeper level of information processing in the current study, over and above the effects from attentional spreading. This independent modulation from attention also suggests that spatial and temporal information of movements, albeit interdependent, might not be fully integrated.

Supplementary Information The online version contains supplementary material available at https://doi.org/10.1007/s10339-022-01078-1.

Acknowledgements The author thanks Thomas Schack for his firm and generous support and Artur Kantarev for assistance in data collection. The author also thanks Theater Bielefeld for supporting the movement recording, and Tiemen Stemerding and Inês Carijó for demonstrating movement sequences.

Funding This research was supported by the Cluster of Excellence Cognitive Interaction Technology 'CITEC' (EXC 277) at Bielefeld University, which has been funded by the German Research Foundation (DFG). Open Access funding enabled and organized by Projekt DEAL.

\section{Declarations}

Conflict of interest The author declares that there is no conflict of interest.

Ethical approval This study was conducted in accordance with the ethical principles stated within the declaration of Helsinki (1964) and was approved by the Ethics Committee of Bielefeld University.

Informed consent Informed consent was obtained from all individual participants included in the study.

Open Access This article is licensed under a Creative Commons Attribution 4.0 International License, which permits use, sharing, adaptation, distribution and reproduction in any medium or format, as long as you give appropriate credit to the original author(s) and the source, provide a link to the Creative Commons licence, and indicate if changes were made. The images or other third party material in this article are included in the article's Creative Commons licence, unless indicated otherwise in a credit line to the material. If material is not included in the article's Creative Commons licence and your intended use is not permitted by statutory regulation or exceeds the permitted use, you will need to obtain permission directly from the copyright holder. To view a copy of this licence, visit http://creativecommons.org/licenses/by/4.0/.

\section{References}

Abend W, Bizzi E, Morasso P (1982) Human arm trajectory formation. Brain 105(Pt 2):331-348. https://doi.org/10.1093/brain/105.2.331

Aly M, Turk-Browne NB (2016) Attention promotes episodic encoding by stabilizing hippocampal representations. Proc Natl Acad Sci 113(4):E420-E429. https://doi.org/10.1073/pnas.1518931113

Benoni H, Tsal Y (2013) Conceptual and methodological concerns in the theory of perceptual load. Front Psychol 4:522. https://doi.org/ 10.3389/fpsyg.2013.00522

Biederman I (1987) Recognition-by-components: a theory of human image understanding. Psychol Rev 94(2):115-147. https://doi.org/ 10.1037/0033-295X.94.2.115

Blake R, Shiffrar M (2007) Perception of human motion. Annu Rev Psychol 58:47-73. https://doi.org/10.1146/annurev.psych.57. 102904.190152

Broadbent DE (1958) Perception and communication. Elsevier

Casasanto D, Boroditsky L (2008) Time in the mind: Using space to think about time. Cognition 106(2):579-593. https://doi.org/10. 1016/j.cognition.2007.03.004

Casasanto D, Fotakopoulou O, Boroditsky L (2010) Space and time in the child's mind: evidence for a cross-dimensional asymmetry. Cogn Sci 34(3):387-405. https://doi.org/10.1111/j.1551-6709. 2010.01094.x

Cavanagh P (1991) Short-range vs long-range motion: not a valid distinction. Spat vis 5(4):303-309. https://doi.org/10.1163/15685 6891x00065

Chen Z (2003) Attentional focus, processing load, and Stroop interference. Percept Psychophys 65(6):888-900. https://doi.org/10. 3758/bf03194822

Chun MM, Turk-Browne NB (2007) Interactions between attention and memory. Curr Opin Neurobiol 17(2):177-184. https://doi.org/10. 1016/j.conb.2007.03.005

Corbetta M, Miezin FM, Dobmeyer S, Shulman GL, Petersen SE (1990) Attentional modulation of neural processing of shape, color, and velocity in humans. Science 248(4962):1556-1559. https://doi.org/10.1126/science. 2360050

Cosman JD, Vecera SP (2012) Object-based attention overrides perceptual load to modulate visual distraction. J Exp Psychol Hum Percept Perform 38(3):576-579. https://doi.org/10.1037/a0027406

de Fockert JW, Rees G, Frith CD, Lavie N (2001) The role of working memory in visual selective attention. Science 291(5509):18031806. https://doi.org/10.1126/science. 1056496

Deutsch JA, Deutsch D (1963) Attention: some theoretical considerations. Psychol Rev 70(1):80. https://doi.org/10.1037/h0039515

Dormal V, Pesenti M (2013) Processing numerosity, length and duration in a three-dimensional Stroop-like task: towards a gradient of processing automaticity? Psychol Res 77(2):116-127. https:// doi.org/10.1007/s00426-012-0414-3

Driver J (2001) A selective review of selective attention research from the past century. Br J Psychol 92(1):53-78. https://doi.org/10. $1348 / 000712601162103$

Duncan J (1980) The locus of interference in the perception of simultaneous stimuli. Psychol Rev 87(3):272-300. https://doi.org/10. 1037/0033-295X.87.3.272 
Faul F, Erdfelder E, Lang A-G, Buchner A (2007) G* Power 3: A flexible statistical power analysis program for the social, behavioral, and biomedical sciences. Behav Res Methods 39(2):175-191. https://doi.org/10.3758/BF03193146

Gazzaley A, Nobre AC (2012) Top-down modulation: bridging selective attention and working memory. Trends Cogn Sci 16(2):129135. https://doi.org/10.1016/j.tics.2011.11.014

Giese MA, Poggio T (2003) Neural mechanisms for the recognition of biological movements. Nat Rev Neurosci 4(3):179-192. https:// doi.org/10.1038/nrn1057

Grahn JA (2012) See what I hear? Beat perception in auditory and visual rhythms. Exp Brain Res 220(1):51-61. https://doi.org/10. 1007/s00221-012-3114-8

Green DM, Swets JA (1966) Signal detection theory and psychophysics. Wiley

Hutmacher F, Kuhbandner C (2020) Detailed long-term memory for unattended, irrelevant, and incidentally encoded auditory information. J Exp Psychol Gen 149(2):222-229. https://doi.org/10. 1037/xge0000650

Jiang Y, Chun MM (2001) Selective attention modulates implicit learning. Quart J Exp Psychol Section A 54(4):1105-1124. https://doi. org/10.1080/713756001

Jiménez L, Mendez C (1999) Which attention is needed for implicit sequence learning? J Exp Psychol Learn Mem Cogn 25(1):236259. https://doi.org/10.1037/0278-7393.25.1.236

Johnston WA, Dark VJ (1986) Selective attention. Annu Rev Psychol 37(1):43-75. https://doi.org/10.1146/annurev.ps.37.020186. 000355

Khetrapal N (2010) Load theory of selective attention and the role of perceptual load: is it time for revision? Eur J Cogn Psychol 22(1):149-156. https://doi.org/10.1080/09541440902787014

Kuhbandner C, Rosas-Corona EA, Spachtholz P (2017) High-fidelity visual long-term memory within an unattended blink of an eye. Front Psychol 8:1859. https://doi.org/10.3389/fpsyg.2017.01859

Lange J, Georg K, Lappe M (2006) Visual perception of biological motion by form: a template-matching analysis. J vis 6(8):836-849. https://doi.org/10.1167/6.8.6

Lavie N (1995) Perceptual load as a necessary condition for selective attention. J Exp Psychol Hum Percept Perform 21(3):451-468. https://doi.org/10.1037/0096-1523.21.3.451

Lavie N (2005) Distracted and confused? Selective attention under load. Trends Cogn Sci 9(2):75-82. https://doi.org/10.1016/j.tics. 2004.12.004

Lavie N (2010) Attention, distraction, and cognitive control under load. Curr Dir Psychol Sci 19(3):143-148. https://doi.org/10.1177/ 0963721410370295

Lavie N, Hirst A, De Fockert JW, Viding E (2004) Load theory of selective attention and cognitive control. J Exp Psychol Gen 133(3):339-354. https://doi.org/10.1037/0096-3445.133.3.339

Luan S, Schooler LJ, Gigerenzer G (2011) A signal-detection analysis of fast-and-frugal trees. Psychol Rev 118(2):316-338. https://doi. org/10.1037/a0022684

Luck SJ, Vogel EK (1997) The capacity of visual working memory for features and conjunctions. Nature 390(6657):279-281. https://doi. org $/ 10.1038 / 36846$

Macmillan NA, Creelman CD (1991) Detection theory: a user's guide. Cambridge University Press

Mayer KM, Vuong QC (2012) The influence of unattended features on object processing depends on task demand. Vision Res 56:20-27. https://doi.org/10.1016/j.visres.2012.01.013
Murphy G, Groeger JA, Greene CM (2016) Twenty years of load theory-where are we now, and where should we go next? Psychon Bull Rev 23(5):1316-1340. https://doi.org/10.3758/ s13423-015-0982-5

Peelen MV, Downing PE (2007) The neural basis of visual body perception. Nat Rev Neurosci 8(8):636-648. https://doi.org/10.1038/ nrn2195

Peirce JW (2007) PsychoPy-psychophysics software in Python. J Neurosci Methods 162(1-2):8-13. https://doi.org/10.1016/j.jneumeth. 2006.11.017

Peirce JW (2008) Generating stimuli for neuroscience using PsychoPy. Front Neuroinform 2(10). https://doi.org/10.3389/neuro.11.010. 2008

Reynolds JH, Chelazzi L (2004) Attentional modulation of visual processing. Annu Rev Neurosci 27:611-647. https://doi.org/10.1146/ annurev.neuro.26.041002.131039

Richard AM, Lee H, Vecera SP (2008) Attentional spreading in objectbased attention. J Exp Psychol Hum Percept Perform 34(4):842853. https://doi.org/10.1037/0096-1523.34.4.842

Santiago J, Román A, Ouellet M (2011) Flexible foundations of abstract thought: a review and a theory. In: Schubert TW, Maass A (eds) Spatial dimensions of social thought. De Gruyter Mouton, pp 39-108. https://doi.org/10.1515/9783110254310.39

Stanislaw H, Todorov N (1999) Calculation of signal detection theory measures. Behav Res Methods Instrum Comput 31(1):137-149. https://doi.org/10.3758/BF03207704

Starr A, Brannon EM (2016) Visuospatial working memory influences the interaction between space and time. Psychon Bull Rev 23(6):1839-1845. https://doi.org/10.3758/s13423-016-1043-4

Thornton IM, Rensink RA, Shiffrar M (2002) Active versus passive processing of biological motion. Perception 31(7):837-853. https://doi.org/10.1068/p3072

Treisman AM (1969) Strategies and models of selective attention. Psychol Rev 76(3):282-299. https://doi.org/10.1037/h0027242

Turk-Browne NB, Jungé JA, Scholl BJ (2005) The automaticity of visual statistical learning. J Exp Psychol Gen 134(4):552-564. https://doi.org/10.1037/0096-3445.134.4.552

Uncapher MR, Rugg MD (2009) Selecting for memory? The influence of selective attention on the mnemonic binding of contextual information. J Neurosci 29(25):8270-8279. https://doi.org/ 10.1523/JNEUROSCI.1043-09.2009

Wood JN (2007) Visual working memory for observed actions. J Exp Psychol Gen 136(4):639-652. https://doi.org/10.1037/0096-3445. 136.4.639

Wood JN (2011) A core knowledge architecture of visual working memory. J Exp Psychol Hum Percept Perform 37(2):357-381. https://doi.org/10.1037/a0021935

Yantis S, Serences JT (2003) Cortical mechanisms of space-based and object-based attentional control. Curr Opin Neurobiol 13(2):187193. https://doi.org/10.1016/s0959-4388(03)00033-3

Publisher's Note Springer Nature remains neutral with regard to jurisdictional claims in published maps and institutional affiliations. 